\title{
Correction to: A new and simple method for measuring in situ field-saturated hydraulic conductivity using a falling-head single cylinder
}

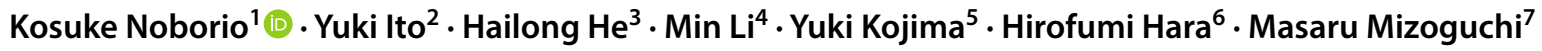

Published online: 13 February 2018

(c) The International Society of Paddy and Water Environment Engineering and Springer Japan KK, part of Springer Nature 2018

\section{Correction to: Paddy Water Environ \\ https://doi.org/10.1007/s10333-017-0617-8}

In the original publication of this article, the equation 12 had a typographical error and has been incorrectly published online. Now the correct equation has been provided in this erratum.

$$
\frac{\mathrm{d} i}{\mathrm{~d} H}=\left|\frac{1}{t+\frac{\beta}{2 \alpha}}\right| .
$$

The original article can be found online at https://doi.org/10.1007/ s10333-017-0617-8.

Kosuke Noborio

noboriok@meiji.ac.jp

1 School of Agriculture, Meiji University, 1-1-1 Higashimita, Tama-ku, Kawasaki, Kanagawa 214-8571, Japan

2 Graduate School of Agriculture, Meiji University, 1-1-1 Higashimita, Tama-ku, Kawasaki, Kanagawa 214-8571, Japan

3 College of Natural Resources and Environment, Northwest A\&F University, Taicheng Rd. 3, Yangling 712100, Shaanxi Province, China

4 Key Laboratory of Agricultural Soil and Water Engineering in Arid and Semiarid Areas, Ministry of Education,

Northwest A\&F University, Yangling 712100,

Shaanxi Province, China
Faculty of Engineering, Gifu University, 1-1 Yanagodo, Gifu City, Gifu 501-1193, Japan

6 Department of Environmental Engineering and Green Technology, Malaysia-Japan International Institute of Technology (MJIIT), Universiti Teknologi Malaysia (UTM), Jalan Sultan Yahya Petra, 54100 Kuala Lumpur, Malaysia

7 Graduate School of Agriculture and Life Sciences, The University of Tokyo, 1-1-1 Yayoi, Bunkyo-ku, Tokyo 113-8657, Japan 